\title{
Prevalence of Intestinal Parasitic Infection in Cancer, Organ Transplant and Primary Immunodeficiency Patients in Tehran, Iran
}

\author{
Abdoulreza Esteghamati ${ }^{1}$, Khadijeh Khanaliha ${ }^{1 *}$, Farah Bokharaei-Salim ${ }^{2}$, \\ Shirin Sayyahfar ${ }^{1}$, Masoomeh Ghaderipour ${ }^{1}$
}

\begin{abstract}
Background: Intestinal parasitic infection in immunodeficient patients especially those with impaired cellular immunity, like neoplasia, renal or heart transplant needs careful consideration. The objective of this study is to evaluate the prevalence of intestinal parasites in different group of patients including cancer patients; organ transplants recipients, and primary immunodeficiency patients. Methods: Stool samples from 190 patients including 80 patients with Primary Immunodeficiency, 85 cancer patients and 25 organ transplant recipients were collected; a direct examination with Phosphate buffered saline (PBS) and formalin ether concentration was performed. The DNA was extracted from parasitologically confirmed patients and nested PCR and sequencing was performed and new obtained sequences of Cryptosporidium parvum and Enterocytozoon bieneusi were compared with deposited ones. Results: In general, the prevalence of parasites was 26/80 (32.5\%) in primary immunodeficiency, 22/85(25.9\%) in cancer group, and 7/25 $(28 \%)$ in organ transplant. The prevalence of intestinal parasitic infections in primary immunodeficiency patients were Blastocystis hominis 13 (16.2\%), Giardia lamblia 10 (12.5\%), Cryptosporidium 1(1.2\%), Chilomastix mesnilii 1 (1.2\%), Dientamoeba fragilis 1(1.2\%). Of 25 organ transplants, 6 (24\%) Cryptosporidium sp were found, all of which were confirmed as Cryptosporidium parvum and one case of Microspora in a heart transplant recipient was confirmed as Enterocytozoon bieneusi by PCR sequencing. The predominant intestinal parasitic infection in cancer patients was 19 (22.3\%) Blastocystis hominis followed by two (2.3\%) Giardia lamblia and one Dientamoeba fragilis 1 (1.1\%). Conclusion: The high rate of infection with Blastocystis hominis was found in cancer patients especially colorectal cancer patients, so careful consideration should be given by physicians. Cryptosporidium $s p$ was found to be the major cause of parasitic intestinal infection in patients with organ transplant compared to primary immunodeficiency patients; so transplant recipients undergoing immunosuppressive therapy should be considered as a risk group for acquiring microsporidiosis and Cryptosporidium infection.
\end{abstract}

Keywords: Immunodeficiency- transplant- cancer- opportunistic- parasite- infection

Asian Pac J Cancer Prev, 20 (2), 495-501

\section{Introduction}

In developing countries, intestinal parasitic infection in immunodeficient patients including, organ transplant recipients, cancer and common variable immunodeficiency patients needs careful consideration. Opportunistic infections are the cause of diarrhea in patients with HIV/AIDS (Khanaliha et al., 2015) and patients under some kind of immunosuppression drugs (Rasti et al., 2017).

Nowadays the increasing use of transplants (kidney, bone marrow, liver, heart, etc.) and the use of new immunosuppressive drugs in patients with cancer and primary autoimmune diseases are observed. (Ferreira and Borges, 2002).
The most frequently reported malignancies were stomach cancer in males and breast cancer in females in Tehran, Iran during 1998 - 2001 (Mohagheghi et al., 2009). Colorectal cancer (CRC) is one the most common cancers worldwide. This cancer is the third most common cancer among Iranian men. According to the results of a study, the incidence of colorectal cancer has increased in Iran during 2003 and 2008 (Rafiemanesh et al., 2016).

It is demonstrated that oxidative stress plays a critical role in the molecular mechanisms of colorectal cancer (CRC) and is shown to be associated with Blastocystis sp, that is the most common intestinal protozoa (Kumarasamy et al., 2017). The pathogenicity of Blastocystis sp. is associated with some disease like acquired immune deficiency syndrome (AIDS) (Fisseha et al., 2017) and 
colorectal cancer (CRC) (Chandramathi et al., 2014). Blastocystis $s p$ in CRC patients during chemotherapy treatment displays opportunistic nature of Blastocystis $s p$ in these patients(Chandramathi et al., 2012).

The second most prevalent primary immunodeficiency is common variable immunodeficiency (CVID) disease. CVID is a combination of humoral and cell-mediated deficiency. It is defined as hypogammaglobulinemia associated with $\operatorname{IgG}$ and $\operatorname{IgA}$ immunoglobulin deficit whereas the immunoglobulin M (IgM) concentration can be normal or low (Chapel and Cunningham-Rundles, 2009). An increased risk of malignancy especially lymphoma and gastric cancer is associated with CVID and malignancy is diagnosed as one of the five clinical CVID phenotypes (Chapel et al., 2008; Mortaz et al., 2016).

Although intravenous immunoglobulin therapy was performed on CVID patients because of the defects of T-cell-mediated, the gastrointestinal disorders were observed (Kalha and Sellin, 2004). The intestinal parasitic infection like Giardia lamblia, Cryptosporidium parvum are common in patients with primary immunodeficiencies (PIDs) patients group (Aguilar et al., 2014).

Parasitic infections rate in organ transplant recipients is unknown, however few patients are symptomatic (Thom and Forrest, 2006). Only 5\% of known human pathogenic parasitic infections which have serious morbidity have been reported in transplant recipients (Barsoum, 2004). The transplant clinicians should be highly suspicious of parasitic infections as an important transmission threat (Barsoum, 2004).

Cryptosporidium is a coccidian intestinal protozoan, which is often found in the human intestine that can cause severe diarrhea in the immunocompromised patient. It is an infamous infection in ileum transplants (Pozio et al., 2004), but has also been reported as a recrudescence disease in recipients of liver (Campos et al., 2000), kidney (Minz et al., 2004), and bone marrow transplants (Müller et al., 2004).Cryptosporidium should be differentiated from other protozoa, Isospora belli and Cyclospora cayetanensis in immunocompromised patients (Verweij and Stensvold, 2014).

The genus Cryptosporidium consist of over 20 species which species like C. parvum from C. hominis are indistinguishable morphologically (Peralta et al., 2016). Cryptosporidium infection in both immunocompromised and immunocompetent individuals may be found by different species and genotypes (Cama et al., 2007).

Microsporidia are intracellular spore-forming protozoa that are present in the environment and can infect the intestine of insects, birds, and mammals. The diagnosis was made by staining and molecular method (Agholi et al., 2013a; Khanaliha et al., 2014). Enterocytozoon bieneusi in patients with HIV disease was reported in several studies (Agholi et al., 2013a).

The aim of this study is to evaluate the prevalence of intestinal parasites in different groups of patients including organ transplants recipients, cancer group and primary immunodeficiency referred to Milad hospital and three general hospitals namely Ali-Asghar, Rasoul-e-Akram hospital, and Rajaie Heart Hospital associated with Iran University of Medical sciences, Tehran, Iran.

\section{Materials and Methods}

This cross-sectional study was performed between July 2016 and November of 2017 including 80 patients with Primary Immunodeficiency, 85 cancer patients and 25 organ transplant recipients who were referred to Milad hospital and three general hospitals namely Ali-Asghar, Rasoul-e-Akram hospitals and Rajaie Heart Hospital associated with Iran University of Medical Sciences, Tehran, Iran.

\section{Stool examination}

Stool samples were collected from 190 patients referred to hospitals associated with Iran University of Medical Sciences and transferred to Research Center of Pediatric Infectious Diseases, Institute of Immunology and Infectious Diseases, Iran University of Medical Sciences. Furthermore, they were examined by direct examination with PBS and then formalin ether concentration was performed and finally examined under microscope with 100x and 400x as final magnification. The modified acid fast staining method was applied for the detection of coccidia parasites infection. The smears were prepared and stained by the modified Ziehl-Neelsen stain. The aniline blue staining method was used to detect spores of microsporidia as described before (Ghaderipour et al., 2017).

\section{DNA extraction}

All of samples were positive for Cryptosporidium and microspora by Ziehl-Neelsen technique and aniline blue staining method respectively were used in PCR. DNA extraction was performed by (Roche Diagnostics GmbH, Mannheim, Germany) according to the manufacturer's instructions

\section{Cryptosporidium}

The nested PCR was performed and an $837 \mathrm{bp}$ fragment amplified of the 18S rRNA gene. The primers were used for nested PCR primers described by Santin et, al (Santín et al., 2006).

5' - T TCTAGAGCTAATACATGCG-3' and 5'-CCCATTTCCTTCGAAACAGGA-3' for primary PCR and 5'-GGAAGGGTTGTATTT-ATTAGATAAAG-3' and 5'-AAGGAGTAAGGAACAACCTCCA-3' for secondary PCR.

PCR was performed in a $25 \mu \mathrm{L}$ mixture containing the template ( $3 \mu \mathrm{L}$ of DNA), $2.5 \mathrm{U}$ of Taq DNA polymerase, $2.5 \mu \mathrm{L}$ of 10x PCR buffer, 20 pmol of each primer, 100 $\mu \mathrm{mol}$ dNTPs, and $0.15 \mathrm{mmol} \mathrm{MgCl}_{2}$. After an initial start at $94^{\circ \mathrm{C}}$ for $3 \mathrm{~min}$, each of cycles consisted of $94^{\circ \mathrm{C}}$ for 45 s, $59^{\circ \mathrm{C}}$ for $45 \mathrm{~s}$ and $72^{\circ \mathrm{C}}$ for 1 min for 35 cycles and ends with $72^{\circ \mathrm{C}}$ for $7 \mathrm{~min}$. The second round was performed by the cycling program consisting of 40 cycles $94^{\circ \mathrm{C}}$ for $30 \mathrm{~s}, 58^{\circ \mathrm{C}}$ for $90 \mathrm{~s}, 72^{\circ \mathrm{C}}$ for $20 \mathrm{~min}$, with an initial at start $94{ }^{\circ \mathrm{C}}$ for $3 \mathrm{~min}$ and ended with $72^{\circ \mathrm{C}}$ for 7 min. Finally, PCR products were electrophoresed on $1 \%$ agarose gel. The positive control was used in each step of PCR and distilled water was used as a negative control. 


\section{Enterocytozoon bieneusi}

The nested PCR was carried out using E. bieneusi specific primers that amplified the ITS region as well as a portion of the flanking large and small subunit ribosomal RNA genes (Santín et al., 2006)

The outer primers were EBITS 3 (5'-GGTCATAGGGATGAAGAG-3') and EBITS4 (5'-TTCGAGTTCTTTCGCGCTC-3'). The inner primers were EBITS1 (5'-GCTCTGAATATCTATGGCT-3') and EBITS2.4 (5'-ATCGCCGACGGATCCAAGTG-3'). Finally, these reactions produced a fragment of $389 \mathrm{bp}$

The reaction mixture $(25 \mu \mathrm{L})$ contained $2.5 \mu \mathrm{L}$ of 10x PCR buffer $0.15 \mathrm{mmol} \mathrm{MgCl} 2,100 \mu \mathrm{mol} d \mathrm{NTPs}, 20$ pmol of each primer, $2.5 \mathrm{U}$ of Taq. The PCR started with denaturation at $94^{\circ \mathrm{C}}$ for $5 \mathrm{~min}, 35$ cycles of amplification (denaturation at $94{ }^{\circ \mathrm{C}}$ for $30 \mathrm{~s}$, annealing at $57^{\circ \mathrm{C}}$ for 30 $\mathrm{s}$, and elongation at $72^{\circ \mathrm{C}}$ for $40 \mathrm{~s}$ ) and then was followed by a final extension at $72^{\circ \mathrm{C}}$ for $10 \mathrm{~min}$. The annealing temperature for the second round of PCR was $55^{\circ \mathrm{C}}$ and other condition was identical with first round of PCR (Santín et al., 2006).

\section{Ethical issues}

The present study was approved by the Ethics Committee of Iran University of Medical Sciences in accordance with the Helsinki Declaration and Guidelines. It also should be mentioned that informed consent was obtained from all patients for the present study.

Sequencing: The second round of PCR was done using inner primers and PCR products were purified by the High Pure PCR Product Purification Kit (Roche Diagnostic, Mannheim, Germany) and were used for direct sequencing using the dye termination method and an ABI 3730xl sequencer (Khanaliha et al., 2017).

The new sequences of Cryptosporidium and E. bieneusi were deposited in GenBank database and compared with deposited sequences by BLAST analysis.

Statistical analysis: Analysis was done using SPSS version 18 (Chicago, IL, USA) and Chi-square test was used to analyze statistical relationship. A statistically significant $\mathrm{P}$ value of $<0.05$ was accepted.

\section{Results}

Overall 190 patient consist of 109 (57.4\%) males and $81(42.6 \%)$ females including 80 CVID and Bruton agammaglobulinemia, 85 cancers group patients: lymphoma cancer, lymphosarcoma, hepatoblastoma, lung

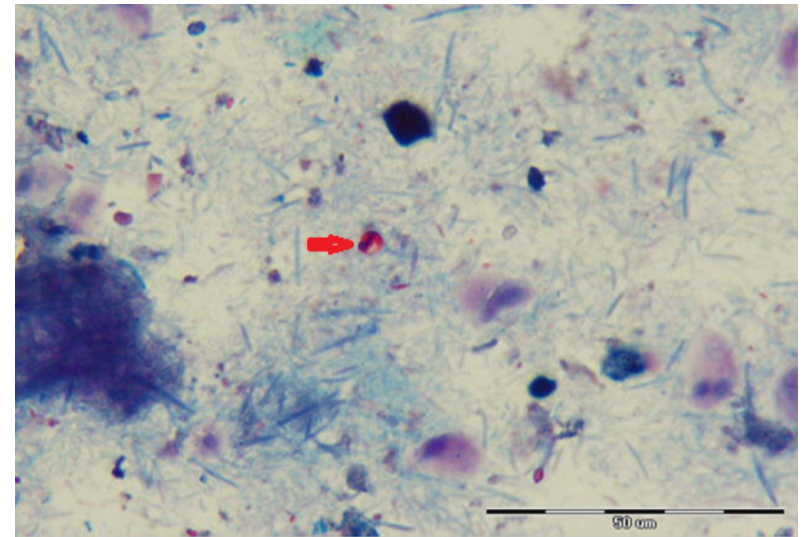

Figure 1. Appearance of Cryptosporidium Oocyst in Stool Samples Collected from Organ Transplant Recipients by Modified Acid Fast Staining Method, 1000x Magnification (Origin Picture).

and colon cancer and 25 organ transplant including liver, kidney and heart were evaluated for parasitic infection

Out of 80 CVID positive patients, 41 (51.2\%) were male and 39 (48.8\%) were female and among 85 cancer group patients, $48(56.5 \%)$ were male and $37(43.5 \%)$ were female and of 25 organ transplant recipients 19 (76\%) were males and $6(24 \%)$ were females. The patient age was between 5-65 years old with the mean age 34 year.

In general, the prevalence of parasites were $26 / 80$ (32.5\%) in primary immunodeficiency, 22/85 (25.9\%) in cancer group, $7 / 25(28 \%)$ in organ transplant. The rate of infection was higher in male $(31.2 \%)$ than female

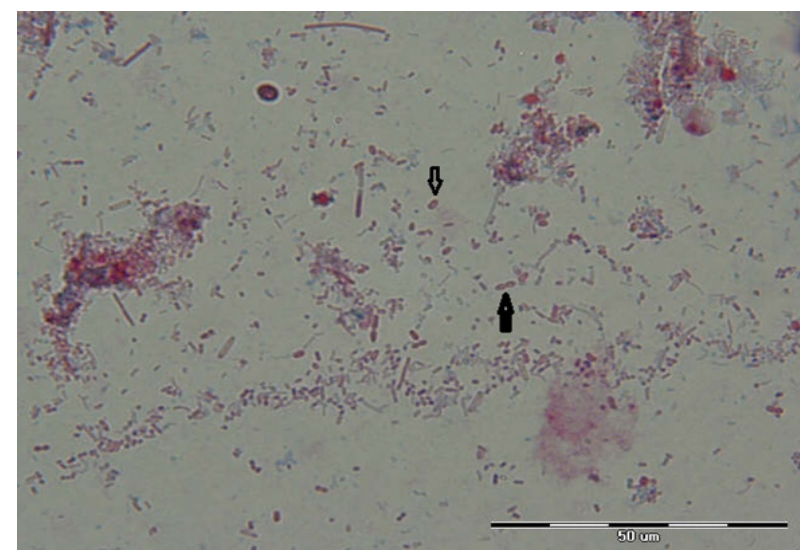

Figure 2. Appearance of E. bieneusi Spores in Stool Samples Collected from Heart Transplant Recipient by Aniline Blue Staining Method, 1,000x Magnification (Origin Picture).

Table 1.Prevalence of Intestinal Parasitic Infection among Different Groups of Patients

\begin{tabular}{lcccccc}
\hline parasites & \multicolumn{2}{c}{ Primary immunodeficiency No: 80} & \multicolumn{2}{c}{ Transplant recipients No:25 } & \multicolumn{2}{c}{ Cancers Patients No:85 } \\
& Male & Female & Male & Female & Male & Female \\
\hline Blastocystis hominis & $6(7.5 \%)$ & $7(8.75 \%)$ & 0 & 0 & $12(14.1 \%)$ & $7(8.2 \%)$ \\
Giardia lamblia & $6(7.5 \%)$ & $4(5 \%)$ & 0 & 0 & $2(2.3 \%)$ & 0 \\
*Cryptosporidium spp. & 0 & $1(1.25 \%)$ & $5(20 \%)$ & $1(4 \%)$ & 0 & 0 \\
Chilomastix mesnilii & 0 & $1(1.25 \%)$ & 0 & 0 & 0 & 0 \\
Dientamoeba fragilis & $1(1.25 \%)$ & 0 & 0 & 0 & $1(1.2 \%)$ & 0 \\
*Microspora & 0 & 0 & $1(4 \%)$ & 0 & 0 & 0 \\
Total & $13(16.25 \%)$ & $13(16.25 \%)$ & $6(24 \%)$ & $1(4 \%)$ & $15(17.6 \%)$ & $7(8.2 \%)$ \\
\hline
\end{tabular}




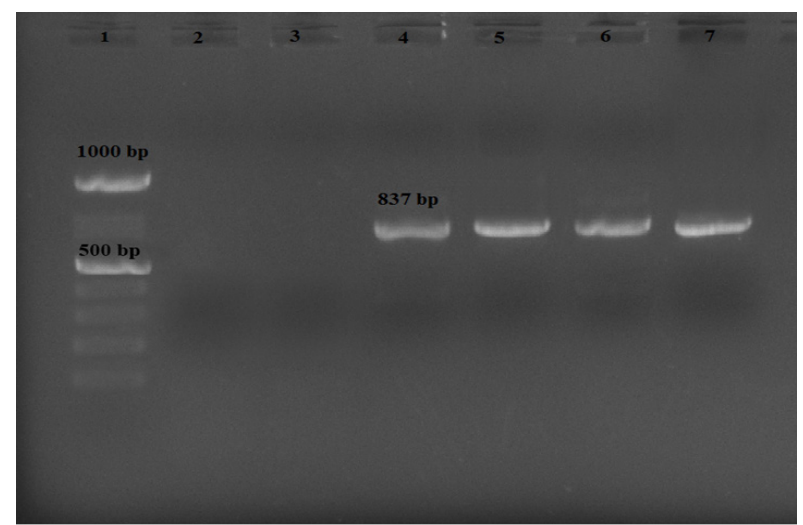

Figure 3. PCR Products of Cryptosporidium in Iranian Transplant Recipients. 1, DNA Ladder Marker; 2, Negative Control; 3, Negative Patient; 4, 5, 6, Positive Patients; 7, Positive Control.

$(18.9 \%)$ cancer patients and also in male $(31.5 \%)$ and female $(16.6 \%)$ OTR patients, but differences were not statistically significant $(\mathrm{P}$ value $=0.1)(\mathrm{P}$ value $=0.4)$. The rate of infection was $(31.7 \%)$ in male and $(33.3 \%)$ in female CVID patients and differences were not statistically significant $(\mathrm{P}$ value $=0.4)$.

The prevalence of intestinal parasitic infection in primary immunodeficiency patients was Blastocystis hominis 13 (16.2\%), Giardia lamblia 10 (12.5\%), Cryptosporidium 1 (1.2\%), Chilomastix mesnilii 1 (1.2\%), Dientamoeba fragilis $1(1.2 \%)$.

Among 85 cancers patients including 39 colorectal cancer and 46 cancers outside gastrointestinal tract (COGT) like lymphoma, lymphosarcoma, hepatoblastoma, lung cancer 19 (22.3\%) Blastocystis hominis and two (2.3\%) Giardia lamblia and one Dientamoeba fragilis 1(1.1\%) were found. Blastocystis hominis were identified in 11/39 (28.2\%) among CRC group and 8/46 (17.4\%) among COGT patients. Although Blastocystis hominis was more common among CRC group patients than COGT ones, the difference between groups wasn't statistically significant $(\mathrm{P}$ value $=0.17)$.

The results of this study show; 6(24\%) Cryptosporidium $s p$ were found in 25 organ transplant recipients including (OTR) including liver, kidney and heart transplant. The oocyst of Cryptosporidium was found in three liver, two kidney and one heart transplant recipients and one additional Cryptosporidium $s p$ was identified in primary immunodeficiency patients by acid fast staining method (Figure 1). One microspora 1 (4\%) was found in a heart transplant recipient using aniline blue staining method (Figure 2). All of patients with cryptosporidiosis and microsporidia infection had diarrhea as a clinical manifestation.

Microspora confirmed as E. bieneusi in a heart transplant recipients by PCR sequencing in this study. The results of prevalence of parasitic infection are summarized in Table 1.

There was no statistically significant difference between the prevalence of parasitic infection in cancer, CVID and OTR patients in this study $(\mathrm{P}$ value $=0.22$; $\mathrm{P}$ value $=0.43)$, however, the difference between Cryptosporidium infection in OTR patients with CVID

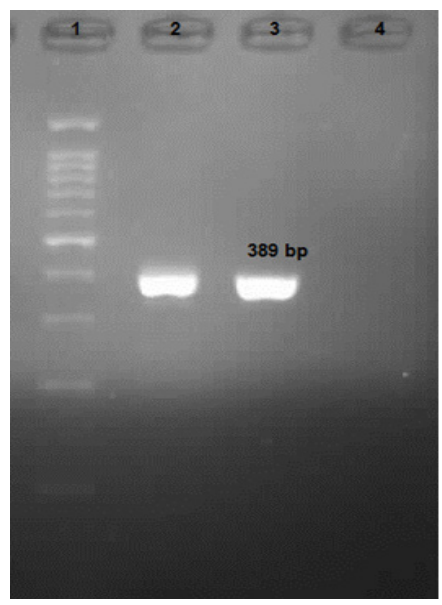

Figure 4. PCR Products of E. bieneusi in Iranian Transplant Recipient; 1, DNA Ladder Marker (100 bp); 2, Positive Control; 3, Positive E. bieneusi Patients (389 bp); 4, Negative Control

and OTR with cancer patients were statistically significant $(\mathrm{P}$ value $=0.0001)$

\section{PCR and Sequencing}

The result of Cryptosporidium PCR is shown in Figure 3.The band of $837 \mathrm{bp}$ fragment of the $18 \mathrm{~S}$ rRNA gene related to Cryptosporidium was amplified from fecal specimens of patients by nested PCR protocol. All PCR positive samples were sequenced to identify the species. The obtained sequences were deposited and compared with other deposited sequences in the GenBank database by BLAST analysis. All of 7 patients infected with Cryptosporidium were also confirmed as C. parvum by PCR sequencing. The isolates had $100 \%$ similarity with the C. parvum genotype 1 (Gen Bank accession no. L16997).

The deposited sequences Gen Bank accession numbers for Cryptosporidium were: MH215510, MH215511, MH215512, MH215513, MH215514, MH215515, and MH215516 in this study.

Enterocytozoon bieneusi was identified in a heart transplant recipient by PCR sequencing. A band of 389 bp was observed in agarose gel electrophoresis of PCR product (Figure. 4). E. bieneusi isolate had 99\% identity with G2-J9, 536 and CHN1 and genotype J in this study. The deposited sequences GenBank accession no for $E$. bieneusi was MH243443.

\section{Discussion}

Parasitic infections are among the most important pathogens that can cause infections in immunocompromised individuals (Ferreira and Borges, 2002). Patients with impaired cellular immunity, like neoplasia, renal or heart transplant patients are at risk of protozoa infection (Ferreira and Borges, 2002).

In this study Blastocystis hominis with the prevalence of $19(22.3 \%)$ was found as the most prevalent parasitic infection among cancers patients. Blastocystis hominis $11 / 39(28.2 \%)$ was detected more frequently in CRC group than COGT patients 8/46 (17.4\%). 
Prevalence of Blastocystis $s p$ is relatively high in developing countries (Tan, 2008) and it was reported as the most common parasites in Iran (Mahni et al., 2016).

The result of a study demonstrated that Blastocystis $s p$. has a significant role in enhancing Azoxymethane (AOM) -induced carcinogenesis damage to the intestinal epithelium and oxidative damage were observed in rats infected with Blastocystis $s p$ and the study proposed that Blastocystis $s p$. is a pathogen protozoa so it is necessary to screen cancer patients for this parasite (Kumarasamy et al., 2017).

The prevalence of Blastocystis $s p$ in CRC patients was $33.3 \%$ in comparison with $25 \%$ in cancers outside gastrointestinal tract (COGT) and $15 \%$ in non-cancer (NC) patients in a study and the result suggested a possible association between subtype-I of Blastocystis hominis and CRC, which could reveal an important role of Blastocystis on CRC condition (Mohamed et al., 2017).

Microsporidiosis has been reported in HIV positive patients but more recently, has been diagnosed in non-HIV-infected individuals (Agholi et al., 2013a; Ghaderipour et al., 2017).

Several studies have reported microsporidial infections in organ transplant recipients, however these infections have not been proved to be transplant-transmitted (Lanternier et al., 2009; Galván et al., 2011). The microsporidiosis have been reported in solid transplant recipients (Carlson et al., 2004), microsporidia were found in kidney recipients (Mohindra et al., 2002; Galván et al., 2011; Visvesvara et al., 2013), pancreas-kidney (Carlson et al., 2004) and heart transplants(Gumbo et al., 1999).

In the present study, E. bieneusi was found in a ten-year-old heart transplant patient who had chronic diarrhea and had received transplant for more than two years. Comparing the new sequence with the result of deposited ones, it was indicated that the $E$. bieneusi isolate had 99\% identity with isolates G2-J9, 536 and CHN1 and genotype $\mathrm{J}$ in this study

Genotype CHN1 was previously detected in human and cattle as the most common genotype and genotype CHN2 was found only in human samples in a study (Zhang et al., 2011).

In a study on among 44 liver transplant children in Shiraz 6.81\% Enterocytozoon bieneusi (genotype D) and $11.36 \%$ C. parvum and C. Meleagridis were identified (Agholi et al., 2013b).

Cryptosporidium infection is more common in overcrowded societies with low degrees of sanitation (Brink et al., 2002) There are few studies on cryptosporidiosis in Iran (Azami et al., 2007). The previous reports indicated the prevalence of this infection in diarrhea patients which was $4.7 \%$ (Sulaiman et al., 2002) and in HIV-patients which was reported 1.5\% (Guyot et al., 2001; Zali et al., 2004).

Giardia lamblia and Cryptosporidium parvum are two parasites that may be found during Primary Immunodeficiency infection like agammaglobulinemia, CVID and Hyper-IgM syndrome with cellular defect, however, among the last cases, Cryptosporidium parvum is more common (Aguilar et al., 2014).

In the present study, Blastocystis hominis 13 (16.2\%), and Giardia lamblia 10 (12.5\%) were the most prevalent parasites especially in primary immunodeficiency patients and one case cryptosporidium parvum 1 (1.2\%) was identified in a patient with CVID who suffered from chronic diarrhea.

Blastocystis hominis (4.2\%) and Giardia lamblia (3.0\%) were also reported as the most prevalent parasites in immunocompromised patients in the previous study (Rasti et al., 2017).

In a study which was performed in Turkey, the most common parasites diagnosed were Cryptosporidium spp, Giardia spp and Blastocystis spp, among CVID patients with clinical manifestation especially diarrhea (Uysal et al., 2016) .

The most common Cryptosporidium infection was found more in organ transplant patients than cancer or patients with primary Immunodeficiency in the present study. All organ transplant recipients had received transplant for more than 1 year. Overall, 7 cases of Cryptosporidium identified that six of them were found in organ transplant recipients including liver, kidney and heart transplant recipients and one additional Cryptosporidium was detected in a CVID patient by acid fast staining. The result of PCR and sequencing show that all of them had $100 \%$ similarity with the C. parvum genotype 1, human genotype (GenBank accession no. L16997) and 99\% similarity with C. parvum strain Human genotype (HFL2) (GenBank accession no. AF093491).

In a study, four Cryptosporidium genotypes in HIV patients were identified: C. parvum genotype 1 (human), C. parvum genotype 2 (bovine), a genotype related to C. felis, and one genotype was similar to a Cryptosporidium $s p$. The corresponding sequences of the C. parvum genotype 1 SSU-rRNA were identical (GenBank accession number L16997) (Pieniazek et al., 1999).

A study which was carried out on immunocompromised Egyptian patients Giardia lamblia with prevalence of $(10 \%)$ showed the most common parasite identified among patients. Other parasites were Cryptospotidium parvum (7\%) Cyclospora cayetanensis (3\%) and Microsporidia species (2\%) (Baiomy et al., 2010).

The presence of $C$ parvum, $C$ hominis, and $C$ meleagridis was confirmed in three patients with primary immunodeficiency by molecular method (Wolska-Kusnierz et al., 2007).

In a study, the 13 Cryptosporidium isolates from immunocompromised patients from Isfahan were evaluated and eight of them were infected with the $C$. parvum genotypes (GenBank accession no. L 16997) (Azami et al., 2007). C. parvum sequences, in the present study, had also $100 \%$ similarity with C. parvum genotypes (GenBank accession no. L 16997)

C. parvum isolates were detected among human and cattle and the L1 subgenotype was the most prevalent and was diagnosed in eight human cases out of nine and in all cattle cases, in a study (Meamar et al., 2007).It has been reported $C$. parvum cattle genotype are predominant among C. parvum isolates from human in some previous study (Azami et al., 2007; Meamar et al., 2007).

Cancer patients especially CRC group had the highest rate of infection with Blastocystis hominis in this study,

Asian Pacific Journal of Cancer Prevention, Vol 20 
so screening of this protozoon for this group of patients with regard to the role of this parasite in oxidative damage to intestinal epithelium is recommended. Furthermore, Blastocystis hominis and Giardia lamblia were the most prevalent parasites in primary immunodeficiency and opportunistic infection like Cryptosporidium and E. bieneusi which were found more in OTR than primary immunodeficiency (CVID and Bruton), so these patients should be considered for opportunistic infection.

Patients with primary Immunodeficiency received azitromycine and were under prophylactic treatment in this study, so this may be a reason for reducing opportunistic infection like microspora and cryptosporidium in this group.

In general patients with impaired T-cell function especially to a CD4 counts of less than $50 / \mathrm{mm}^{3}$, whether it is inherited or acquired immunocompromised disease or patients under treatment depending on degrees of immune-suppression, are at higher risk of Cryptosporidium and microspora infection (Hunter and Nichols, 2002).

Overall, immunodeficient patients with clinical manifestation and impaired cellular immunity and under treatment of immune suppression should be considered for opportunistic parasitic infection.

In conclusion, the high rate of infection with Blastocystis hominis was found in cancer patients especially CRC patients, so careful consideration should be given by physicians. Cryptosporidium parvum was found to be the major cause of parasitic intestinal infection in patients with organ transplant compared to other CVID patients, so organ transplant recipients undergoing immunosuppressive treatment should be considered a risk group for acquiring microsporidia and Cryptosporidium infection.

\section{Ethical considerations}

This study was approved by the Ethics Committee of Iran University of Medical Sciences code number (IR.IUMS.REC1395-02-131-28109) in accordance in accordance with Helsinki Declaration and Guidelines. It also should be mentioned that informed consent has been obtained from all human participants.

\section{Sources of funding}

This study was funded by Research Center of Pediatric Infectious Diseases, Iran University of Medical Sciences in Tehran, Iran with Grant number (95-02-131-28109).

\section{Conflicts of interest}

The authors declare that they have no conflicts of interest.

\section{References}

Agholi M, Hatam GR, Motazedian MH (2013a). HIV/AIDS-associated opportunistic protozoal diarrhea. AIDS Res Hum Retroviruses, 29, 35-41.

Agholi M, Hatam GR, Motazedian MH (2013b). Microsporidia and coccidia as causes of persistence diarrhea among liver transplant children: incidence rate and species/genotypes.
Pediatr Infect Dis J, 32, 185-7.

Aguilar C, Malphettes M, Donadieu J, et al (2014). Prevention of infections during primary immunodeficiency. Clin Infect Dis, 59, 1462-70.

Azami M, Moghaddam DD, Salehi R, et al (2007). The identification of Cryptosporidium species in Isfahan, Iran by PCR-RFLP analysis of the $18 \mathrm{~S}$ rRNA gene. Mol Biol, 41, 851-6.

Baiomy A, Mohamed K, Ghannam M, et al (2010). Opportunistic parasitic infections among immunocompromised Egyptian patients. J Egypt Soc Parasitol, 40, 797-808.

Barsoum RS (2004). Parasitic infections in organ transplantation. Exp Clin Transplant, 2, 258-67.

Brink A-K, Mahe C, Watera C, et al (2002). Diarrhoea, CD4 counts and enteric infections in a community-based cohort of HIV-infected adults in Uganda. $J$ Infect, 45, 99-106.

Cama VA, Ross JM, Crawford S, et al (2007). Differences in clinical manifestations among Cryptosporidium species and subtypes in HIV-infected persons. J Infect Dis, 196, 684-91.

Campos M, Jouzdani E, Sempoux C, et al (2000). Sclerosing cholangitis associated to cryptosporidiosis in liver-transplanted children. Eur J Pediatr, 159, 113-5.

Carlson JR, Li L, Helton CL, et al (2004). Disseminated microsporidiosis in a pancreas/kidney transplant recipient. Arch Pathol Lab Med, 128, 41-3.

Chandramathi S, Suresh K, Anita ZB, et al (2012). Infections of Blastocystis hominis and microsporidia in cancer patients: are they opportunistic?. Trans R Soc Trop Med Hyg, 106, 267-9.

Chandramathi S, Suresh K, Sivanandam S, et al (2014). Stress exacerbates infectivity and pathogenicity of Blastocystis hominis: in vitro and in vivo evidences. PLoS One, 9 , e94567.

Chapel H, Cunningham-Rundles C (2009). Update in understanding common variable immunodeficiency disorders (CVIDs) and the management of patients with these conditions. Br J Haematol, 145, 709-27.

Chapel H, Lucas M, Lee M, et al (2008). Common variable immunodeficiency disorders: division into distinct clinical phenotypes. Blood, 112, 277-86.

Ferreira MS, Borges AS (2002). Some aspects of protozoan infections in immunocompromised patients: a review. $\mathrm{Mem}$ Inst Oswaldo Cruz, 97, 443-57.

Fisseha B, Petros B, Woldemichael T, et al (1999). Diarrhoea-associated parasitic infectious agents in AIDS patients within selected Addis Ababa Hospitals. Ethiop $J$ Health Dev, 13,169-74

Galván A, Sánchez AM, Valentín MP, et al (2011). First cases of microsporidiosis in transplant recipients in Spain and review of the literature. J Clin Microbiol, 49, 1301-6.

Ghaderipour M, Khanaliha K, Mohebali M, et al (2017). Emerging intestinal microsporidia infection in general population in Jiroft district, southeastern Iran: A cross-sectional study in 2013-2014. Iran J Public Health, 46, 1697.

Gumbo T, Hobbs RE, Carlyn C, et al (1999). Microsporidia infection in transplant patients. Transplantation, 67, 482-4.

Guyot K, Follet-Dumoulin A, Lelievre E, et al (2001). Molecular characterization of Cryptosporidium isolates obtained from humans in France. J Clin Microbiol, 39, 3472-80.

Hunter PR, Nichols G (2002). Epidemiology and clinical features of Cryptosporidium infection in immunocompromised patients. Clin Microbiol Rev, 15, 145-54.

Kalha I, Sellin JH (2004). Common variable immunodeficiency and the gastrointestinal tract. Curr Gastroenterol Rep, $\mathbf{6}$, 377-83.

Khanaliha K, Masoumi-Asl H, Bokharaei-Salim F, et al (2017). Double-stranded RNA viral infection of Trichomonas 
vaginalis (TVV1) in Iranian isolates. Microb Pathog, 109, 56-60.

Khanaliha K, Mirjalali H, Mohebali M, et al (2014). Comparison of three staining methods for the detection of intestinal Microspora spp. Iran J Parasitol, 9, 445-51.

Khanaliha K, Mohebali M, Davoudi S, et al (2015). Detection of emergence Cyclospora cayetanensis in A HIV+/AIDS patient with diarrhea from Tehran: a case report. Iran $J$ Public Health, 44, 865-8.

Kumarasamy V, Kuppusamy UR, Jayalakshmi P, et al (2017). Exacerbation of colon carcinogenesis by Blastocystis $s p$. PLoS One, 12, e0183097.

Lanternier F, Boutboul D, Menotti J, et al (2009). Microsporidiosis in solid organ transplant recipients: two Enterocytozoon bieneusi cases and review. Transpl Infect Dis, 11, 83-8.

Mahni MB, Rezaeian M, Eshrat Beigom K, et al (2016). Prevalence of intestinal parasitic infections in Jiroft, Kerman province, Iran. Iran J Parasitol, 11, 232-8.

Meamar A, Rezaian M, Mohraz M, et al (2007). A comparative analysis of intestinal parasitic infections between $\mathrm{HIV}+/$ AIDS patients and non-HIV infected individuals. Iran $J$ Parasitol, 2, 1-6.

Minz M, Udgiri NK, Heer MK, et al (2004). Cryptosporidiasis in live related renal transplant recipients: a single center experience. Transplantation, 77, 1916-7.

Mohagheghi MA, Mosavi-Jarrahi A, Malekzadeh R, et al (2009). Cancer incidence in tehran metropolis: the first report from the tehran population-based cancer registry. Arch Iran Med, 12, 15-23.

Mohamed AM, Ahmed MA, Ahmed SA, et al (2017). Predominance and association risk of Blastocystis hominis subtype I in colorectal cancer: a case control study. Infect Agent Cancer, 12, 21.

Mohindra A, Lee M, Visvesvara G, et al (2002). Disseminated microsporidiosis in a renal transplant recipient. Transpl Infect Dis, 4, 102-7.

Mortaz E, Tabarsi P, Mansouri D, et al (2016). Cancers related to immunodeficiencies: update and perspectives. Front Immunol, 7, 365

Müller CI, Zeiser R, Grüllich C, et al (2004). Intestinal cryptosporidiosis mimicking acute graft-versus-host disease following matched unrelated hematopoietic stem cell transplantation. Transplantation, 77, 1478-9.

Peralta RHS, Velásquez JN, Cunha FdS, et al (2016). Genetic diversity of Cryptosporidium identified in clinical samples from cities in Brazil and Argentina. Mem Inst Oswaldo Cruz, 111, 30-6.

Pieniazek NJ, Bornay-Llinares FJ, Slemenda SB, et al (1999). New Cryptosporidium genotypes in HIV-infected persons. Emerg Infect Dis, 5, 444-9.

Pozio E, Rivasi F, CACCIS (2004). Infection with Cryptosporidium hominis and reinfection with Cryptosporidium parvum in a transplanted ileum. Apmis, 112, 309-13.

Rafiemanesh H, Pakzad R, Abedi M, et al (2016). Colorectal cancer in Iran: Epidemiology and morphology trends. EXCLI $J, \mathbf{1 5}, 738-44$.

Rasti S, Hassanzadeh M, Hooshyar H, et al (2017). Intestinal parasitic infections in different groups of immunocompromised patients in Kashan and Qom cities, central Iran. Scand J Gastroenterol, 52, 738-41.

Santín M, Trout JM, Vecino JAC, et al (2006). Cryptosporidium, Giardia and Enterocytozoon bieneusi in cats from Bogota (Colombia) and genotyping of isolates. Vet Parasitol, 141, 334-9.

Sulaiman IM, Lal AA, Xiao L (2002). Molecular phylogeny and evolutionary relationships of Cryptosporidium parasites at the actin locus. $J$ Parasitol, 88, 388-94.
Tan KS (2008). New insights on classification, identification, and clinical relevance of Blastocystis spp. Clin Microbiol Rev, 21, 639-65.

Thom K, Forrest G (2006). Gastrointestinal infections in immunocompromised hosts. Curr Opin Gastroenterol, 22, 18-23.

Uysal S, Tunali V, Öztürk EA, et al (2016). Incidence of parasitic diarrhea in patients with common variable immune deficiency. Turkiye Parazitol Derg, 40, 67-71.

Verweij JJ, Stensvold CR (2014). Molecular testing for clinical diagnosis and epidemiological investigations of intestinal parasitic infections. Clin Microbiol Rev, 27, 371-418.

Visvesvara GS, Arrowood MJ, Qvarnstrom Y, et al (2013). Concurrent parasitic infections in a renal transplant patient. Emerg Infect Dis, 19, 2044-5.

Wolska-Kusnierz B, Bajer A, Caccio S, et al (2007). Cryptosporidium infection in patients with primary immunodeficiencies. J Pediatr Gastroenterol Nutr, 45, 458-64.

Zali MR, Mehr AJ, Rezaian M, et al (2004). Prevalence of intestinal parasitic pathogens among HIV-positive individuals in Iran. Jpn J Infect Dis, 57, 268-70.

Zhang X, Wang Z, Su Y, et al (2011). Identification and genotyping of Enterocytozoon bieneusi in China. J Clin Microbiol, 49, 2006-8.

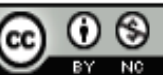

This work is licensed under a Creative Commons AttributionNon Commercial 4.0 International License. 